\title{
X-ray properties of a sample of NLS1 selected from the SDSS
}

\author{
N. Castelló-Mor* \\ Instituto de Física de Cantabria (CSIC-UC) \\ Avda. Los Castros s/n (Edif. Juan Jordá), E 39005, Santander (Spain) \\ E-mail: castellodifca.unican.es
}

\section{Barcons}

Instituto de Física de Cantabria (CSIC-UC)

Avda. Los Castros s/n (Edif. Juan Jordá), E 39005, Santander (Spain)

E-mail: barconsdifca.unican.es

\section{Ballo}

Instituto de Física de Cantabria (CSIC-UC)

Avda. Los Castros s/n (Edif. Juan Jordá), E 39005, Santander (Spain)

E-mail: ballodifca.unican.es

\section{F.J. Carrera}

Instituto de Física de Cantabria (CSIC-UC)

Avda. Los Castros s/n (Edif. Juan Jordá), E 39005, Santander (Spain)

E-mail: carreraflifca.unican.es

Galaxy surveys have revealed the existence of Narrow Emission Line Galaxies (NELG) which are spectroscopically classified as starforming (SF), but whose X-ray luminosity exceeds $10^{42} \mathrm{erg} \mathrm{s}^{-1}$ which unambiguously signals the presence of an AGN. The origin for such a discrepancy is not understood. In order to addres this issue, we present a study using a large sample of 239 NELG $\left(\operatorname{FWHM}\left(H_{\beta}\right)<1200 \mathrm{~km} / \mathrm{s}\right)$ from the SDSS, for which we can construct the BPT diagram and that are detected in the hard $(2-10 \mathrm{keV}) \mathrm{X}$-ray band within the $2 \mathrm{XMM} \mathrm{X}$-ray source catalogue. We find that 33 of these objects are diagnosed as starforming galaxies but their X-ray luminosity exceeds $10^{42} \mathrm{erg} \mathrm{s}^{-1}$. We conducted a detailed analysis of this population, using other optical spectral features, like the FWHM of the $H_{\beta}$ emission line and the X-ray spectral properties. We find that the bulk of this misclassified population, which represents over $10 \%$ of our full sample of hard X-ray detected NELGs, is constituted by NLS1 galaxies.

Narrow-Line Seyfert 1 Galaxies and their place in the Universe (NLS1),

Milan (Italy)

April 04-06, 2011

\footnotetext{
*Speaker.
} 


\section{Introduction}

Determining the fraction of galaxies hosting AGN at their centers is an essential step for studies of galaxy evolution. The way AGN are usually identified among the large samples of galaxies in massive optical spectroscopic surveys is almost invariably through their emission lines. The signature of the emission lines in galaxies convey information about the emitting source such as the power and nature of the underlying exciting source, what allows one to classify the sources depending on their physical property nature, like starforming (SF) or hosts of Active Galactic Nucleus (AGN). The underlying idea is that the emission lines in normal SF galaxies are powered by massive stars, so there is an upper limit on the intensities ratio of the collisionally excited lines ([OIII],[NII],[SII] among others) with respect to the recombination lines (such as $\mathrm{H}_{\alpha}$ or $\mathrm{H}_{\beta}$ ). Unlike this mechanism, the photons from AGN extend to higher energies than those of the massive stars and therefore, they induce more heating, implying that optical collisionally excited lines will be brighter with respect to recombination lines than in the case of ionization by massive stars only. Baldwin et al. (1981) were amongst the first to introduce robust emission line diagnostic ratios able to distinguish between starforming processes and active nuclear emission [1]. These and other diagrams were later used by Veilleux \& Osterbrock (1987) to derive a semi-empirical classification scheme to distinguish between AGN and SF galaxies [14]. Kewley et al. (2001) used these same diagrams to derive a purely theoretical classification scheme [8]. Kauffmann et al. (2003) and Stasinśka et al. (2006) later extended this scheme (see [7][11] for more information). However, line diagnostics might not be fully reliable in detecting AGN among galaxy samples due to the fact that emission lines can be hidden, diluted or contaminated by stellar galaxy light (see e.g. [10][9][4][[13]).

When X-ray data and optical spectra are available for the same galaxies, it occasionally happens that galaxies which are optically diagnosed as starforming, exhibit large X-ray luminosities, in excess to that of the most luminous SF galaxies in the local Universe by over an order of magnitude $\left(>10^{42} \mathrm{erg} \mathrm{s}^{-1}\right.$ ), where such X-ray emission can only be due to accretion onto a super-massive black hole). The origin for such a discrepancy is not understood. Trouille \& Barger (2010) compared the optical classification with the X-ray properties of a complete sample in three Chandra deep fields [12]. They found that the optical diagnostic diagram misidentifies $20-50 \%$ of their $\mathrm{X}$-ray selected AGN, which are misclassified as SF galaxies. They postulate that such objects have a suppressed [OIII] emission due to the complexity of the structure of the narrow line region, which causes many ionizing photons from the AGN not to be absorbed. the aim of this paper is to explore the origin of this possible misidentification in a large nearby sample where information on both optical emission line data and X-ray spectral properties can be best detemined than in distant samples.

\section{Optical and X-ray properties}

The sample is composed by NELG from SDSS (DR7) in which the involved emission lines in the BPT diagram ([OIII] $\lambda 5007$, [NII] $\lambda 6584, \mathrm{H}_{\alpha}$ and $\mathrm{H}_{\beta}$ ) have been detected. We have excluded sources which have no X-ray conterpart on the 2XMMi catalogue (DR3). The BPT diagram has 


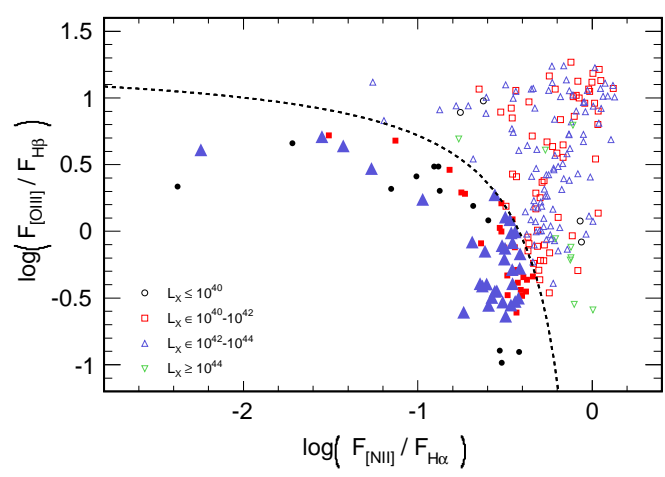

Figure 1: Emission line diagnostic diagram (BPT diagram) for the whole sample of NELG. The curve separating the AGN from the non-AGN (filled marks) zone is from Kauffmann et al. (2003). The points change in form and in color according to X-ray $(2-10 \mathrm{keV})$ luminsoty range.

been used to infer whether the gas in a galaxy is heated by star formation or radiation from an accretion disk around a central SMBH. To be conservative, we have adopted the borderline between SF and AGN galaxies presented by Kauffmann et al. (2003) ([7], hereafter Kauff03). Following this criterion we can directly obtain an optical classification for our sources: 168 out of these 239 NELG have been classified as AGN and the remaining 71 as SF. If we focus on SF population we find that 33 of them (hereafter Missing-AGN sample) are hosting an AGN since their $2-10 \mathrm{keV}$ $\mathrm{X}$-ray luminosities are over $10^{42} \mathrm{erg} \mathrm{s}^{-1}$ (triangles up under the line in Figure 1). If confirmed as non-AGN, then these luminous X-ray sources will be the most powerful starburst galaxies known, exceeding the X-ray luminosity of the brightest starforming galaxies in the local Universe.

Having discussed the optical classification of our sample of NELG and noting that there appear to be mismatches with the X-ray luminosities of some of our objects, we present a comparison of their optical and X-ray properties through three parameters which are able to provide us clues to understand the nature and to characterize the Missing-AGN sample. In order to gain zeroth-order X-ray spectral information we have used a Hardness Ratio parameter $(H R)^{1}$; on the other hand, the Thickness parameter $(T)^{2}$ and $X$-ray-to-optical flux ratio $(X / O)^{3}$ were used to evaluate the $\mathrm{X}$-ray absorption and to discriminate between different classes of X-ray sources (see [2][3][5] and [6] for more information about these three parameters). According to the typical values for both kinds of sources, we expect that $T$ as well as $X / O$ by SF galaxies to fall in $0.1 T<1$ and $X / O<0.1$; moreover we expect that AGN display $T \sim 0.1-100$ and $X / O>0.1$. One would also expect that SF galaxies have an X-ray spectrum dominated by a thermal component which is softer than the typical power-law spectra exhibited by AGN which is harder. However, looking at the population of NELG as classified through the BPT diagram, we cannot define a range of values that isolates AGN

\footnotetext{
${ }^{1} H R \equiv(H-S) /(H+S)$, where $H$ and $S$ are the PSF and vignetting corrected count rates in the $0.5-2 \mathrm{keV}$ and $2-4.5 \mathrm{keV}$ energy bands, respectively.

${ }^{2}$ The thickness parameter represents the ratio between the $2-10 \mathrm{keV}$ observed flux (corrected for Galactic absorption) and the reddening-corrected flux of the $[\mathrm{OIII}] \lambda 5007$ emission line.

${ }^{3}$ For this work, we have defined the X-ray-to-optical flux ratio using the observed X-ray flux in the $0.5-4.5 \mathrm{keV}$ energy range and the optical red-band flux, $f_{\text {band }}\left[\mathrm{erg} / \mathrm{cm}^{2} / \mathrm{s}\right]=10^{-\frac{m_{A B}+48.6}{2.5}} \times \frac{d v}{v} \times \frac{c}{\lambda_{\text {filter }}}$.
} 

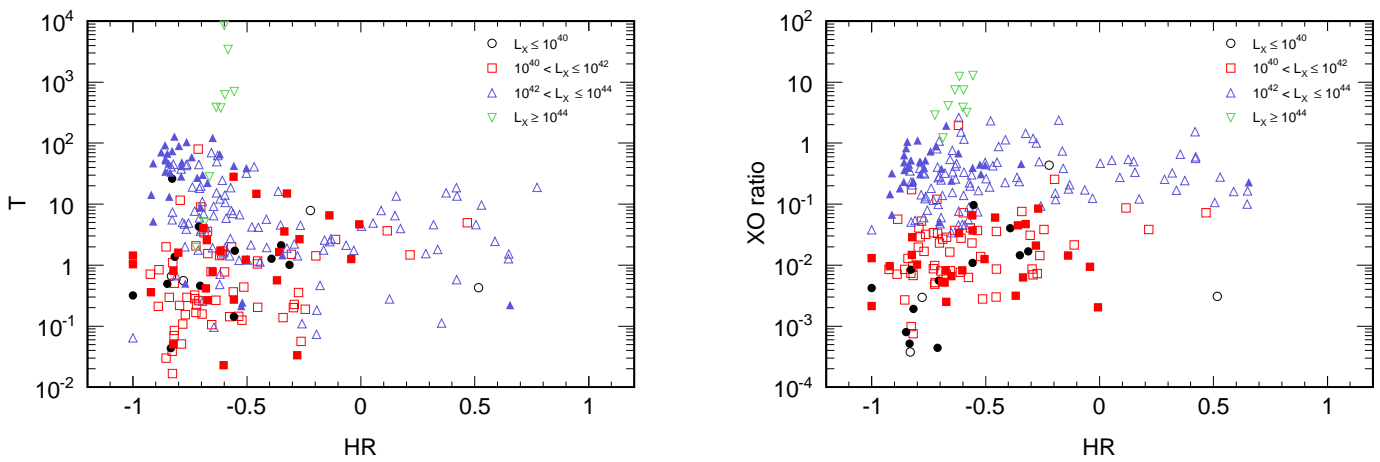

Figure 2: Optical and X-ray properties. Left panel: thickness parameter vs. $H R$ for the NELG sample. Right panel: distribution in X-ray-to-optical flux ratio as a function of $H R$. Different symbols mark X-ray luminosity ranges and the filled/empty symbols represent the optical classification according to Kauff03 (SF/AGN).

from the rest of the sources neither using $H R, T$ or $X / O$ values: NELG classified as SF or AGN galaxies do not occupy a definite region in this parameter space (see Figure 2). In principle the mix of optically diagnosed SF and AGN galaxies sitting below $T<0.1$ and/or $X / O<0.1$ do not show a clear trend in their hardness ratios. Nevertheless, if we split up the whole sample in two populations according to Kauff03, we find that the $\log T$ as well as the $\log X / O$ distributions display a bimodal shape (see Figure 2) for both populations. Only in the case of optically-classified SF, this bimodal feature is directly linked to the values of $\mathrm{H}_{\beta}$ FWHM: all the sources of the Missing-AGN sample exhibit an $\operatorname{FWHM}\left(\mathrm{H}_{\beta}\right) \gtrsim 400 \mathrm{~km} / \mathrm{s}$ and the remaining of SF galaxies display smaller values. The high values for both parameters $(T$ and $X / O)$, two orders of magnitude higher than the average in starforming galaxies but typical of Seyfert 1, the low measured $H R$ and the extreme values for $\mathrm{H}_{\beta}$ FWHM are consistent with these high X-ray luminosities NELGs optically diagnosed as SF galaxies, being Narrow-Line Seyfert 1 candidates (NLS1).

To test this hypothesis we conducted an X-ray spectral analysis for all sources of this Missing$A G N$ sample. The X-ray spectral properties of this population appear to be fairly uniform. The spectra are well reproduced by the combination of blackbody or partial covering and steep powerlaw components. Despite their optical lines consistent with a SF galaxy, signs of AGN activity have been detected in X-rays as a steep spectra $\left(\Gamma_{2-10 \mathrm{keV}} \gtrsim 2\right)$ and extra emission below $\sim 2 \mathrm{keV}$, which support the presence of an AGN with the typical X-ray spectral shape of NLS1.

\section{Conclusion}

The most remarkable result of this comparison, BPT diagram- $L_{X}$, is that about $10 \%$ (Missing$A G N$ sample) of the overall NELG sample would be classified as starforming galaxies according to Kauff03 but display signs of AGN activity in the X-ray band.

We therefore conclude that these AGN missed by the BPT diagram turn out to be Narrow-Line Seyfert 1 with very small broad-line widths, $400 \mathrm{~km} / \mathrm{s} \lesssim \operatorname{FWHM}\left(H_{\beta}\right) \lesssim 1200 \mathrm{~km} / \mathrm{s}$, which have a high accretion rate $(X / O>0.1, T>1)$ and these objects exhibit a soft $\mathrm{X}$-ray excess wherever 
there is enough S/N (27/33); furthermore, all these 27 sources, with very narrow lines, show strong FeII blends, which is not observed in starforming galaxies.

\section{Acknowledgments}

Partial financial support for this work was provided by the spanish Ministry of Science and Innovation, through grant AYA2009-08059.

\section{References}

[1] J.A. Baldwin, M.M. Phillips and R. Terlevich 1981, Classification parameters for the emission-line spectra of extragalactic objects, PASP, 935

[2] L. Bassani. et al. 1999, A Three-dimensional Diagnostic Diagram for Seyfert 2 Galaxies: Probing X-Ray Absorption and Compton Thickness, ApJ, 121473

[3] K. Beuermann et al. 1999, Identification of soft high galactic latitude RASS X-ray sources. II. Sources with PSPC count rate $C R<0.5 \mathrm{cts} / \mathrm{s}$ A\&A,347 47

[4] A. Caccianigaet al. 2007, Elusive AGN in the XMM-Newton bright serendipitous survey, A\&A, 470 557

[5] Della Ceca, R. et al. 2004, Exploring the X-ray sky with the XMM-Newton bright serendipitous survey, $\mathrm{A} \& \mathrm{~A}, 428383$

[6] F. Fiore et al. 2003, The HELLAS2XMM IdF survey: on the nature of high X-ray to optical flux ratio sources, [arXiv:astro-ph/0310298]

[7] G. Kauffmann et al. 2003, The host galaxies of active galactic nuclei, MNRAS, 3461055

[8] L.J. Kewley et al. 2001, The host galaxies of active galactic nuclei, ApJ, 556121

[9] M.J. Page et al. 2006, X-ray spectra of sources in the 13H XMM-Newton/Chandra deep field, MNRAS, 369156

[10] P. Severgnini et al. 2003, XMM-Newton observations reveal AGN in apparently normal galaxies, A\&A, 406483.

[11] G. Stasińska, et al. 2006, Semi-empirical analysis of Sloan Digital Sky Survey galaxies - III. How to distinguish AGN hosts, MNRAS, 371972

[12] L. Trouille and A.J. Barger, 2010, The OPTX Project. IV. How Reliable is [O III] as a Measure of AGN Activity?, IOP, 722 212, [arXiv: 1008 .1582]

[13] J.R. Trump et al. 2009, The Nature of Optically Dull Active Galactic Nuclei in COSMOS, IOP, 706 797, [arXiv:0910.2672]

[14] S. Veilleux and D.E. Osterbrock, 1987, Spectral classification of emission-line galaxies, STI, 63295 